\title{
3-D areal shot-source modeling
}

\author{
Yang Qiqiang \\ Department of Mathematics, Qiongzhou college, Sanya, China \\ keeponmoving@126.com
}

Keywords: 3-D, modeling, areal shot-source, Hartley transform,.

\begin{abstract}
A program for 3-D modeling using areal-shot source to solve the scalar wave equation is presented, implemented and tested. The method developed here in which the wave field is advanced in time by using standard time differencing schemes utilizes a spatial numerical grid to calculate spatial derivatives by the fast Hartley transform. The Hartley transform which avoids computational redundancies in terms of the number of operations and memory requirements is more efficient and economical than its progenitor. These features are crucial when dealing with 3-D seismic data. The method can save at least a magnitude of computations. The proposed algorithm can be easily implemented in parallel architecture.
\end{abstract}

\section{Introduction}

The addition of a third dimension to seismic data acquisition offers significant improvement of our reflection images of geologic structures, but potentially at a substantial increase in data processing cost involving large data volumes and extensive computational facilities. Though the increasing availability of computer power had made 3D seismic data processing feasible, it is still difficult to provide 3-D seismic data to testify 3-D migration or velocity analysis methods for excessive computations. Recent 3-D modeling methods just focused on the simulation of seismic responses. Muti and Fou(1989) presented 3-D finite difference method. Another approach to modeling is pseudospectral method which uses Fourier transforms and requires fewer grid points per wavelength than other numerical modeling methods such as the finite-difference and finite-element methods to achieve the same accuracy. The characteristics of pseudospectral algorithms are described by references[1,2,3]. As the required storage extremely exceeded the capacity of the central memory at that time, the algorithm called for retrieving and restoring 3-D data sets in each of time steps. Jing Wen,George, A.McMechan[4] also developed a pseudospectral scheme dealing with 3-D data with the limitation that the velocity was constant. In most applications the Hartley transform can be an attractive and replacement for the well known complex Fourier transform. Further, the Hartley transform can be obtained from the Fourier transform by replacing the exponential kernel exp(-iux) by $\cos (u x)+\sin (u x)$. Both direct and inverse transforms possess the same kernel, unlike the Fourier transform, and hence there is no need to keep track of $+i$ and $-i$ versions for identifying the direct and inverse transforms. The Hartley transform is an attractive alternative to the Fourier transform in 3-D modeling wherein a large amount of data is handled.

Berkhout [5] presented areal shot record migration method. In his approach the point shot records are synthesized into an areal source response prior to shot-record migration, an enormous data reduction can be obtained. The resulting areal shot record can be migrated using the same scheme as the one for conventional shot-record migration. The main advantage of areal shot-record migration is that the extrapolations need not be done for all the individual shot records, but for the areal shot record only. This makes the areal shot record approach very attractive for the processing of 3-D data. This paper generalized the idea of synthesized areal shot-source to modeling method. The property of the solution of wave equation claims the rightness of the method. In this paper, we start with an introduction to modeling by a Hartley method. Next, areal shot-source is used in modeling. Finally, results are shown. 


\section{3-D modeling by a Hartley method}

When the density is constant, the acoustic wave equation reads

$$
\frac{\partial^{2} p}{\partial x^{2}}+\frac{\partial^{2} p}{\partial y^{2}}+\frac{\partial^{2} p}{\partial z^{2}}+S=\frac{1}{v^{2}} \frac{\partial^{2} p}{\partial t^{2}},
$$

where $P=P(x, y, z, t)$ represents pressure, $v(x, y, z)$ represents the velocity and $S(x, y, z, t)$ represents the source item which equals the divergence of the body forces.

From the wave field given at $t$ and $t-\Delta t$, we approximate its values at time $t+\Delta t$ by the expression

$$
P(t+\Delta t) \approx 2 P(t)-P(t-\Delta t)+v^{2}\left(\frac{\partial^{2} P}{\partial x^{2}}+\frac{\partial^{2} P}{\partial y^{2}}+\frac{\partial^{2} P}{\partial z^{2}}+S\right) \Delta t^{2} .
$$

The space derivative terms in Eq.2 are computed by implementing

$$
\begin{aligned}
& \frac{\partial^{2} P(n \Delta t)}{\partial x^{2}}+\frac{\partial^{2} P(n \Delta t)}{\partial y^{2}}+\frac{\partial^{2} P(n \Delta t)}{\partial z^{2}}=\sum_{k_{x}} \sum_{k_{y}} \sum_{k_{z}}-\left(k_{x}^{2}+k_{y}^{2}+k_{z}^{2}\right) P\left(k_{x}, k_{y}, k_{z}, n \Delta t\right) \\
& \cdot \operatorname{cas}\left(x k_{x}\right) \operatorname{cas}\left(y k_{y}\right) \operatorname{cas}\left(z k_{z}\right) .
\end{aligned}
$$

in which $P\left(k_{x}, k_{y}, k_{z}, n \Delta t\right)$ is the three-dimension Hartley transform of $P(x, y, z, n \Delta t)$ with respect to variables $x, y$ and $z$. First, a fast Hartley transform is performed on $P(x, y, z, t)$ with respects to variables $x, y$ and $z$. The result $P\left(k_{x}, k_{y}, k_{z}, t\right)$ is then multiplied by $-\left(k_{x}{ }^{2}+k_{y}{ }^{2}+k_{z}{ }^{2}\right)$. This operation is followed by an inverse fast Hartley transform into the spatial domain yielding the last result. To keep the algorithm stable, $\left[(1 / \Delta x)^{2}+(1 / \Delta y)^{2}+(1 / \Delta z)^{2}\right]^{1 / 2} v \Delta t \leq 2 / \pi$ is required.

\section{Areal shot source modeling}

In frequency domain, 3-D Helmholtze equation operator reads:

$L=\frac{\partial^{2}}{\partial x^{2}}+\frac{\partial^{2}}{\partial y^{2}}+\frac{\partial^{2}}{\partial z^{2}}-\frac{\omega^{2}}{v^{2}}$,

where $\omega$ denotes angular frequency, it is easy to testify $L$ has the following properties:

if $L P_{k}=S_{k}(\mathrm{k}=1,2, \ldots, \mathrm{K})$, where $s_{k}=s_{k}\left(x, y, z_{0}, \omega\right)$ represents the source function, $z_{0}$ represents the surface, $P_{k}=P_{k}(x, y, z, \omega)$ represents the pressure function. The source record $p_{k}\left(x, y, z=z_{0}, \omega\right)$ is the function value of $P_{k}$ at the surface and is the solution of Helmholtze equation of forward problem.

(1) Linearity: $L \sum_{k=1}^{K} a_{k} P_{k}=\sum_{k=1}^{K} a_{k} S_{k}$;

(2) Shifting: $L P_{k}\left(t-t_{1}\right)=S_{k}\left(t-t_{1}\right)$.

In the frequency domain after Hartley transform:

$L\left[P_{k}(\omega) \cos \left(\omega t_{1}\right)+P_{k}(-\omega) \sin \left(\omega t_{1}\right)\right]=S_{k}(\omega) \cos \left(\omega t_{1}\right)+S_{k}(-\omega) \sin \left(\omega t_{1}\right)$.

The source vector of a single point source (dipole) at the surface reads:

$$
S_{i, j}\left(z_{0}\right)=S^{\prime}(\omega)\left[\begin{array}{l}
0, \ldots, 0,0,0, \ldots, 0 \\
\ldots \ldots \ldots \ldots \ldots \ldots . . \\
0, \ldots, 0,1,0, \ldots, 0 \\
\ldots \ldots \ldots \ldots \ldots \ldots . . . \\
0, \ldots, 0,0,0, \ldots ., 0
\end{array}\right],
$$

where the only nonzero value of the vector in the ith row and jth column indicates the position of the dipole, and $S^{\prime}(\omega)$ is the source signature. However, source vector $S_{i, j}\left(z_{0}\right)$ can be used for any kind of source wave field. For areal shot- source, we may write 


$$
S\left(z_{0}\right)=\left[\begin{array}{l}
S_{1,1}^{\prime}(\omega), S_{1,2}^{\prime}(\omega), \ldots, S_{1, N Y}^{\prime}(\omega) \\
S_{2,1}^{\prime}(\omega), S_{2,2}^{\prime}(\omega), \ldots, S_{2, N Y}^{\prime}(\omega) \\
\ldots \ldots \ldots \ldots \ldots \ldots \ldots \ldots \ldots \ldots \ldots \ldots \ldots \ldots \ldots \ldots \ldots \ldots \ldots \ldots \ldots \ldots \ldots \ldots \\
S_{N X-1,1}^{\prime}(\omega), S_{N X-1,2}^{\prime}(\omega), \ldots, S_{N X-1, N Y}^{\prime}(\omega) \\
S_{N X, 1}^{\prime}(\omega), S_{N X, 2}^{\prime}(\omega), \ldots, S_{N X, N Y}^{\prime}(\omega)
\end{array}\right]
$$

Assume the areal source wavefield has a single wavefront with a space-invariant signature with $t_{i, j}=p_{x}\left(x_{k}-x_{c}\right)+p_{y}\left(y_{j}-y_{c}\right)$ and $p_{x}=\sin \alpha / v_{0}, p_{y}=\sin \beta / v_{0}$,where $\alpha$ and $\beta$ are incidence angles of the source wavefield at $\left(x_{i}, y_{j}\right)$ along $x$ axis and $y$ axis, respectively, $x_{c}$ and $y_{c}$ represent the lateral positions with zero time shift.

Let $P=\sum_{i=1}^{N X} \sum_{j=1}^{N Y}\left(P_{i, j}(\omega) \cos \left(\omega t_{i, j}\right)+P_{i, j}(-\omega) \sin \left(\omega t_{i, j}\right)\right), \quad S=\sum_{i=1}^{N X} \sum_{j=1}^{N Y}\left(S_{i, j}(\omega) \cos \left(\omega t_{i, j}\right)+S_{i, j}(-\omega) \sin \left(\omega t_{i, j}\right)\right)$. With property (1) and (2),

$$
\begin{aligned}
& L P=L \sum_{i=1}^{N X} \sum_{j=1}^{N Y}\left(P_{i, j}(\omega) \cos \left(\omega t_{i, j}\right)+P_{i, j}(-\omega) \sin \left(\omega t_{i, j}\right)\right)=\sum_{i=1}^{N X} \sum_{j=1}^{N Y} L\left(P_{i, j}(\omega) \cos \left(\omega t_{i, j}\right)+P_{i, j}(-\omega) \sin \left(\omega t_{i, j}\right)\right) \\
& =\sum_{i=1}^{N X} \sum_{j=1}^{N Y}\left(S_{i, j}(\omega) \cos \left(\omega t_{i, j}\right)+S_{i, j}(-\omega) \sin \left(\omega t_{i, j}\right)\right)=S
\end{aligned}
$$

is obtained. Then the rightness of both the proposed modeling method and the areal shot-record migration method is claimed.

An areal shot-source shown by Eq.7 is applied to Eq.1, and then an areal shot-record is obtained that is the same with $P$ shown by Eq.7 by stepping using Eq.2 and Eq.3. To avoid boundary reflections, an absorbing boundary as a 20-point weighting function[6] is applied to $P$ on the bottom and lateral edges of the spatial grid.

\section{Computer implementation and example}

The following example shows the result and feasibility of the method. The physical model has a central symmetrical structure with three flat layers and two anticlines as shown by Fig.1a. The calculation used $256 \times 256 \times 400$ mesh points with grid spacing of $25 \mathrm{~m}$ and $5 \mathrm{~m}$ in the $x, y$ and $z$ directions and was carried to 4500 time steps with time step $0.4 \mathrm{~ms}$.

Fig.1b and Fig.1c show two of areal shot-sources adopted with ray parameters (39.6us/m, 0us/m) and $(-59.3 \mathrm{us} / \mathrm{m}, 0 \mathrm{us} / \mathrm{m})$, respectively. Fig.2 a-e display the snapshots of the wave field with ray parameters $(39.6 \mathrm{us} / \mathrm{m}, 0 \mathrm{us} / \mathrm{m})$ at different time steps. From the figure, we can see that since the absorbing boundary has been applied on the lateral edges and the bottom of the 3-D volume the energy has been attenuated to be very weak near boundaries and little energy has penetrated through the boundaries. The recorded data corresponding to the above areal shot-sources is shown by Fig.2f and Fig.2g.

\section{Summary}

3-D areal shot-source modeling yields a record that is the same as the synthesized areal-shot record in areal shot-record migration using the same ray parameters. In this way the extrapolations need not be done for all individual shot-source, but for one areal shot-source. So the computations time become tolerable and thus efficiently provides data for testing 3-D areal shot-record migration and 3-D residual migration velocity analysis. And the resulted Hartley algorithm is efficient in computation time and memory requirements. The example shows that the method is practicable and attractive. 


\section{Acknowledgements}

This research was supported by Hainan Natural Science Foundation (Grant No. 113008).

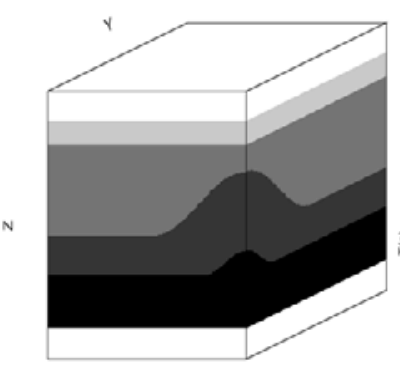

a

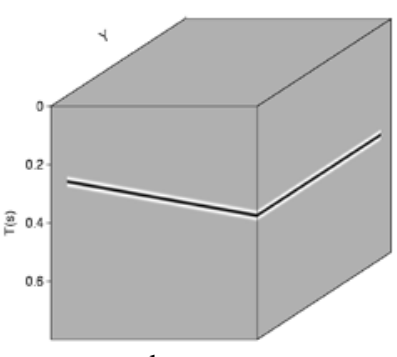

b

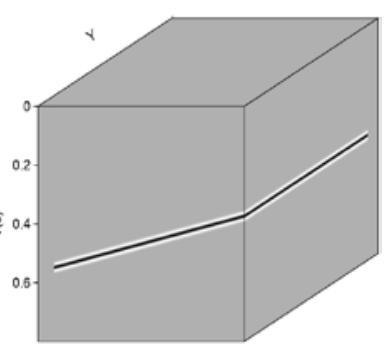

C

Fig. 1. (a) Velocity model with three flat layers and two anticlines. The velocities of the layers from the top to the bottom are $2100 \mathrm{~m} / \mathrm{s}, 2700 \mathrm{~m} / \mathrm{s}, 3500 \mathrm{~m} / \mathrm{s}, 4100 \mathrm{~m} / \mathrm{s}, 4600 \mathrm{~m} / \mathrm{s}, 2100 \mathrm{~m} / \mathrm{s}$, respectively; (b) An areal shot-source with ray parameters (39.6us $/ \mathrm{m}, 0 \mathrm{us} / \mathrm{m})$; (c) An areal shot-source with ray parameters (-59.3us $/ \mathrm{m}, 0 \mathrm{us} / \mathrm{m})$.

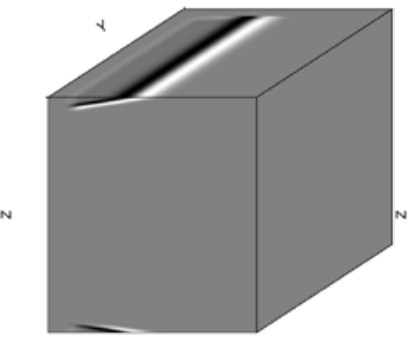

$\mathrm{at}=0.3 \mathrm{~s}$

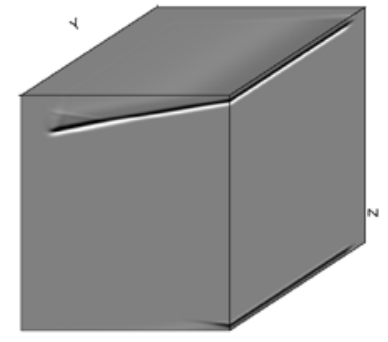

$\mathrm{b} \mathrm{t}=0.4 \mathrm{~s}$

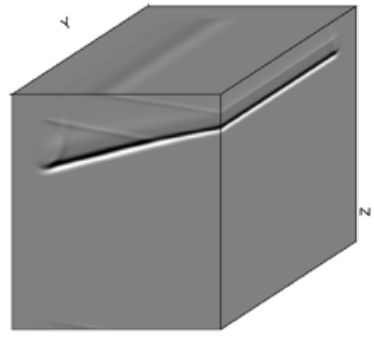

$\mathrm{ct}=0.5 \mathrm{~s}$

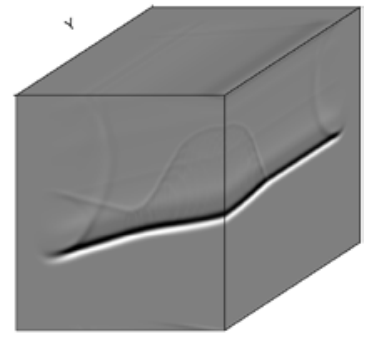

$\mathrm{d} t=0.7 \mathrm{~s}$

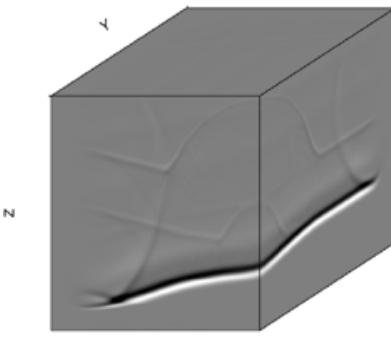

e $\mathrm{t}=0.8 \mathrm{~s}$

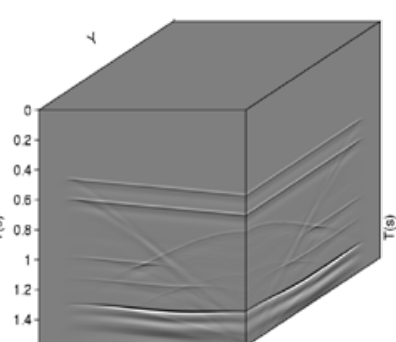

f

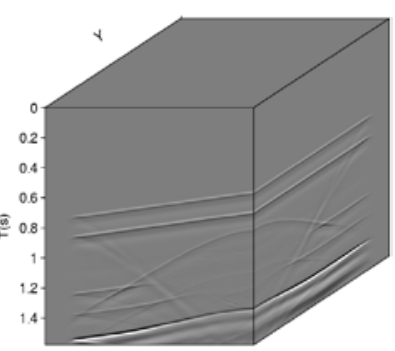

Fig. 2. (a-e) Time sequence showing the evolution of the wave from the areal shot-source shown in Fig. 1b;

$(f, g)$ Reflected waves recorded and the synthesized record at the surface $z=0$.

\section{References}

[1] Daudt, C. R., Braile, L. W., Nowack, R. L., and Chiang, C. S.. A comparison of finite-difference and Fourier method calculations of synthetic seismograms. Bull. Seis. Soc. Am., 79 (1989),1210-1230.

[2] Dan D.Kosloff, and Edip Baysal,Forward modeling by a Fourier method: Geophysics,47 (1982),1402-1412.

[3] Moshe Reshef, Dan Kosloff, Mickey Edwards,and Chris Hsiung, Three-dimensional acoustic modeling by the Fourier method: Geophysics,53 (1988),1175-1183.

[4] Jing Wen,George A. McMechan,and Michael W.Booth,Three-dimensional modeling and migration of seismic data using Fourier transforms:Geophysics,53 (1988),1194-1201.

[5] Berkhout,A.J.,1992,Areal shot record technology:J.Seis.Expl.,1,no.3. Geophysics,47 (1982),1402-1412.

[6] Cerjan, C. , Kosloff. D., Kosloff. R. and Reshef. M.. A nonreflecting boundary condition for discrete acoustic and elastic wave equation. Geophysics, 50 (1985),705-708. 